\title{
Diagnostic challenges and surgical management of co-existent osteoblastoma and aneurysmal bone cyst of the lumbar spine in a child: case report
}

\author{
Kaunda Ibebuike ${ }^{1}$, Mark Roussot ${ }^{2}$, James Watt ${ }^{2}$, \\ Garret Skead $^{3}$, Robert Dunn²
}

1. Visiting Spine Fellow, Division of Neurosurgery, Department of Surgery, Imo State University Teaching Hospital, Orlu, Nigeria.

2. Department of Orthopaedic Surgery, Groote Schuur Hospital, Cape Town, South Africa.

3. Division of Anatomical Pathology, University of Cape Town and National Health Laboratory Service, Groote Schuur Hospital, Cape Town, South Africa.

\begin{abstract} patient. roimaging studies and histopathological assessment of resected tissue. fusion.

DOI: https://dx.doi.org/10.4314/ahs.v19i2.56 abs.v19i2.56

\section{Introduction}

Osteoblastomas (OTB) are uncommon and benign tumours of osteoblastic differentiation and constitute less than $1 \%$ of primary bone tumours. ${ }^{1-3}$ They frequently affect the spine, which accounts for 30 to $40 \%$ of all osteoblastomas occurring in the second to third decades of life,
\end{abstract}

Background: Osteoblastomas (OTB) with co-existent aneurysmal bone cysts (ABC) of the spine are extremely rare and more so in the lumbar spine. To the authors' knowledge, only one case of lumbar spine involvement has been reported in a paediatric

Objectives: To highlight diagnostic challenges and surgical management of co-existent lumbar OTB-ACB in a child.

Methods: We present a 14-year old female with low back pain and progressive swelling of 2 months. She was evaluated by neu-

Results: X-ray showed a lytic lesion in L3 with loss of the left pedicle. Magnetic resonance imaging (MRI) revealed a fluid-filled, heterogeneously enhanced, lobulated lesion posterior to the vertebral bodies of L3, L4 and L5, which infiltrated the quadratus lumborum muscles bilaterally, and histologically diagnosed as ABC. The L3 pedicle also revealed an expanded, heterogeneously enhanced mass lesion which appeared to be different and showed no fluid-filled levels, histologically diagnosed as OTB. Surgical intervention involved tumour excision with partial spondylectomy of L3 vertebra, posterior instrumented stabilization and

Conclusion: The case highlights the diagnostic challenges of co-existent OTB-ABC and the significant role of surgical management via spinal reconstruction, stabilization and fusion after gross total tumour excision.

Keywords: Osteoblastoma, aneurysmal bone cyst, lumbar vertebra, paediatric, instrumented fusion.

Cite as: Ibebuike K, Roussot M, Watt J, Skead G, Dunn R. Diagnostic challenges and surgical management of co-existent osteoblastoma and aneurysmal bone cyst of the Lumbar spine in a paediatric patient: case report. Afri Health Sci.2019;19(2): 2294-2301. bttps:/ / dx.doi.org/10.4314/

\section{Corresponding author:}

Kaunda Ibebuike,

Division of Neurosurgery

Department of Surgery

Imo State University Teaching Hospital,

Orlu 473212

Imo State, Nigeria

Phone: +2348063409555

Email: ibebuikeke@yahoo.com

with a 2:1 male-to-female predominance. ${ }^{3-7}$ Spinal osteoblastoma is rare in the lumbar spine compared to cervical spine involvement (20-40\%) and most often arise within the posterior elements, while vertebral body involvement rarely occurs, especially in isolation. ${ }^{3,4,8}$ Patients characteristically complain of dull localized pain with associated paraesthesias, paraparesis and paraplegia, depending on the extent of associated compression of neural structures. ${ }^{6,7}$ The radiographic presentation displays a well-circumscribed lesion within the cortical bone, which is characteristically larger than $2 \mathrm{~cm}$ in diameter with some $50 \%$ being radiolucent, and $20 \%$ osteoblastic in appearance. ${ }^{6,7}$ The lesion is separated from surrounding soft tissue by a peripheral thin and sclerotic shell and does not have an intense, reactive zone of bone surrounding it, in contrast to osteoid osteoma. ${ }^{6,7}$ The general histologic features of osteoblastoma are characterized by cellular osteoblastic African (C) 2019 Ibebuike et al. Licensee African Health Sciences. This is an Open Access article distributed under the terms of the Creative commons Attribution License (https://creativecommons.org/licenses/BY/4.0), which permits unrestricted use, distribution, and reproduction in any medium, provided the original work is properly cited. 
tissue with active intercellular production of osteoid material and primitive woven bone; fibrovascular stroma and in $89 \%$ of cases there is minimal or no mitotic activity. ${ }^{6,7}$

An aneurysmal bone cyst (ABC) is also uncommon and benign, a locally proliferative osseous disorder of non-neoplastic nature which constitutes $1.4 \%$ of all primary bone tumours in children and adults, with a slight female preponderance. ${ }^{9}$ ABCs occur less often in the spine (10-15\%) compared to long bones, but it is even rarer in the lumbar spine. ${ }^{9-11} \mathrm{ABC}$ of the spine typically presents as a paravertebral soft tissue mass with associated local pain as the most common symptom. The pain is characteristically worse at night and on lying down. Vertebral deformity such as scoliosis and kyphosis are occasionally caused by these tumours, and neurological deficits may occur. The radiographic presentation is characterized by multiloculated lesion with fluid-fluid levels, which may reveal contrast enhancement of the periosteal rim and septations on MRI. ${ }^{7,12}$ These histologic features of ABC reveal that primary $\mathrm{ABC}$ has fibrous-walled channels that have complete or incomplete lining of endothelial cells, but with no elastic lamina or muscular layers, unlike true blood vessels; whereas secondary $\mathrm{ABC}$ has the same microscopic characteristics, but with the added findings of a co-existing lesion which could be benign or malignant. Although both OTB and ABC have been described sep- arately in previous literatures, few reports have described ABC co-existent with OTB. The combination of osteoblastoma and aneurysmal bone cyst may be seen in $10-15 \%$ of cases of primary bone neoplasm, but rare in the lumbar spine. ${ }^{7,10}$ To the authors' knowledge, only one case in a child has been reported. ${ }^{12}$ We report a case of osteoblastoma with co-existent aneurysmal bone cyst in the lumbar spine of a 14-year-old child, to highlight the diagnostic challenges and the surgical intervention with spinal reconstruction, stabilization and fusion.

\section{Case report}

A 14-year old female was referred from a provincial hospital to our Spinal Unit with low back pain with radiation to the legs, and progressive swelling over the lower back for 2 months. There was associated weakness in the lower limbs, mild loss of weight but no night sweats, fever, cough or a known TB contact, and no bowel and bladder dysfunction. There was no known past medical history of malignancy or infection with human immunodeficiency virus (HIV). The physical examination showed an asthenic young female with no significant peripheral lymphadenopathy. Her vital signs were normal and her Glasgow coma scale (GCS) was 15/15. She had a normal gait but struggled with heel-heel walking. There was a large mildly tender 15 x $10 \mathrm{~cm}$ firm mass over the lumbar spine with a normal overlying skin, and no obvious spinal deformity (Fig. 1).

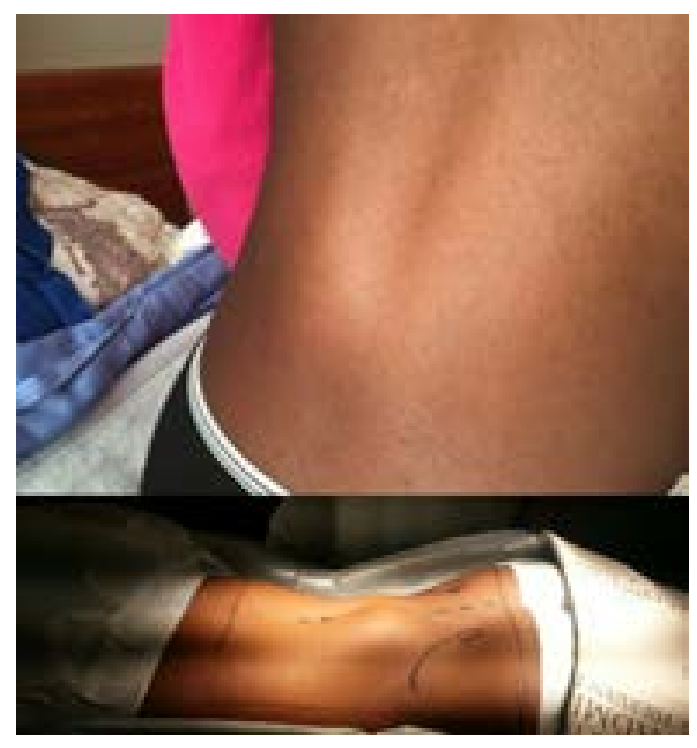

Figure 1. Firm $15 \times 10 \mathrm{~cm}$ mass over lumbar spine and patient positioning/skin marking for tumour resection, L2-L4 pedicle screw fixation and fusion. 
There was normal sensorimotor function in the upper limbs but reduced power at L5 and S1 supply in the lower limbs bilaterally (power $4 / 5$ ). The deep tendon reflexes were essentially normal, and other systems were essentially without any abnormality. X-ray showed a lytic lesion in L3 as well as loss of the left pedicle (Fig. 2).

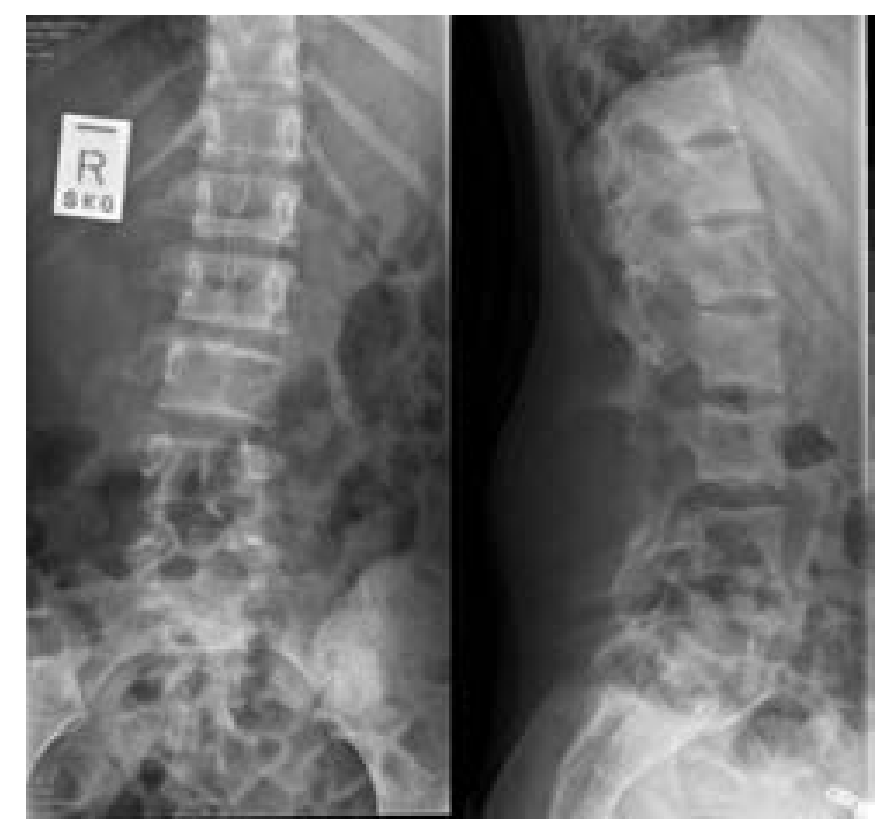

Figure 2. Anteroposterior and lateral plain lumbar spine X-rays showing lytic lesion in L3 as well as loss of the left pedicle ((absent) pedicle sign also known as the winking owl sign).

MRI revealed a lobulated lesion posterior to the vertebral bodies of L3, L4 and L5 with the epi centre of the lesion to be the posterior elements of L3 (Fig. 3). It also revealed an ivory vertebral body at L3 with an expanded and heterogeneously contrast enhancing lesion at the left pedicle of L3. There was associated heterogeneously enhancing muscle lesion with fluid-fluid levels. The lesion infiltrated the quadratus lumborum muscles bilaterally, posterior to the vertebral bodies of L3, L4 and L5. The mass lesion on the L3 pedicle looked different and showed no fluid-fluid levels (Fig. 3). 


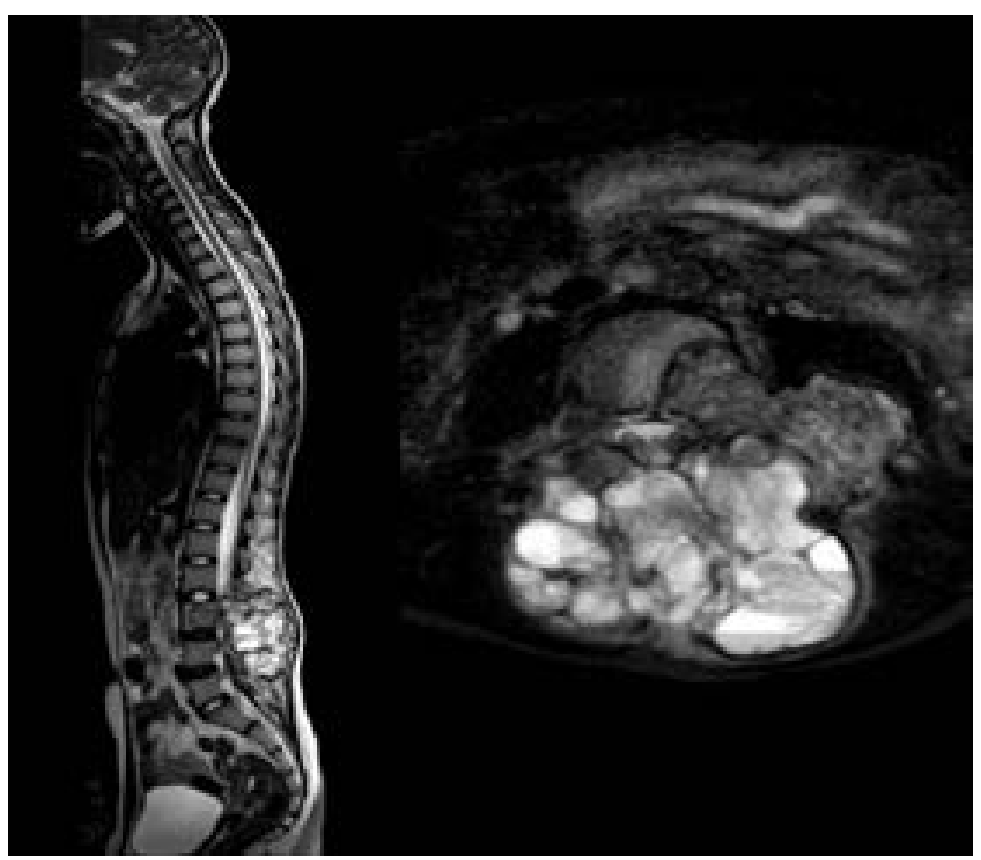

Figure 3. Sagittal and axial lumbar T2-weighted MRI showing lobulated lesion posterior to the vertebral bodies of L3, L4 and L5 with the epicentre of the lesion to be the posterior elements of L3. Note also a different mass lesion in the L3 pedicle which showed no fluid-fluid levels, and a mass lesion in the muscles which appears to have fluidfluid levels.

There was no fracture but there was bony expansion, and the lesion did not extend into the spinal canal. There was extension to involve the psoas muscle on the left, which appeared expanded and heterogeneous. There was also heterogeneous enhancement of the left psoas muscles and the quadratus lumborum. The radiological differential diagnosis on MRI were that of a mass lesion with features suggestive of aneurysmal bone cyst, giant cell tumour, chondroblastoma or osteoblastoma. The tumour was excised aimed at achieving en bloc resection with wide surgical margins and avoidance of tumour breach; partial spondylectomy of affected L3 vertebra, and posterior instrumented stabilization and fusion. The surgical technique involved initial dissection to identify tissue planes, extent of paraspinal muscle involvement and spinal bony involvement. This revealed total involvement and destruction of the posterior elements of L3 with extension to the L3 left pedicle and the L3 spinal canal. During the dissection, the tumour capsule was breached and we also encountered massive primary haemorrhage, which together with the extension of the tumour into the spinal canal altered our aim to achieve en bloc resection. She received massive blood transfusion intraoperatively using both autologous blood transfusion (through the use of cell saver), and homologous blood transfusion. The tumour was excised with wide surgical margins down to the exposed thecal sac. Thereafter pedicle screw instrumentation into L2 and L4 was performed before the final excision of tumour remnants, followed by partial spondylectomy of the affected side of L3 vertebra. Stabilization of the spinal segments was then finalized with rods and screws and a cross-linkage to both rods. Fusion was performed using fibular strut allograft cut to fit into the L3 spondylectomy, and to fuse L2-L4 after discectomy of adjoining L2-L3 and L3-L4 intervertebral discs. A split humeral allograft between the L2 and L4 spinous processes was also used to aid in reconstruction of the posterior tension band between L2 to L4 (Fig. 4). 


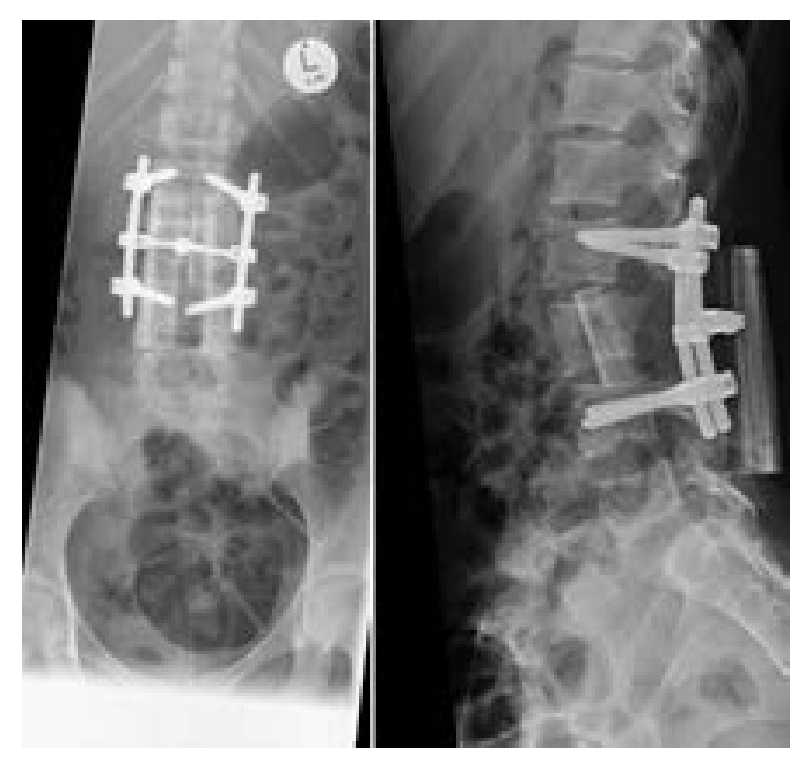

Figure 4. Post-operative anteroposterior and lateral plain X-rays of the same patient after tumour resection, L2-L4 pedicle screw stabilization and fusion with fibular/humeral strut allografts.

Histopathology evaluation of the biopsy specimen demonstrated two distinct components. One component was characterized by cystic, blood-filled spaces, as seen in aneurysmal bone cyst (Fig. 5). In addition, there were solid areas with osteoblastic differentiation, comprising plump, round to spindled osteoblast-like cells interspersed between delicate, lace-like trabeculae of osteoid and maturing woven bone (Fig. 5). The solid component contained numerous osteoclast-like giant cells and scattered larger, cytologically atypical cells (Fig. 5). Mitotic activity was prominent, but atypical mitotic figures were absent. The lesion had a permeative edge, with extension into surrounding soft tissue. The amount of osteoid was variable, showing only focal apposition with the atypical cells, and for the most part demonstrating haphazard distribution within the solid sheets of smaller osteoblast-like cells. 


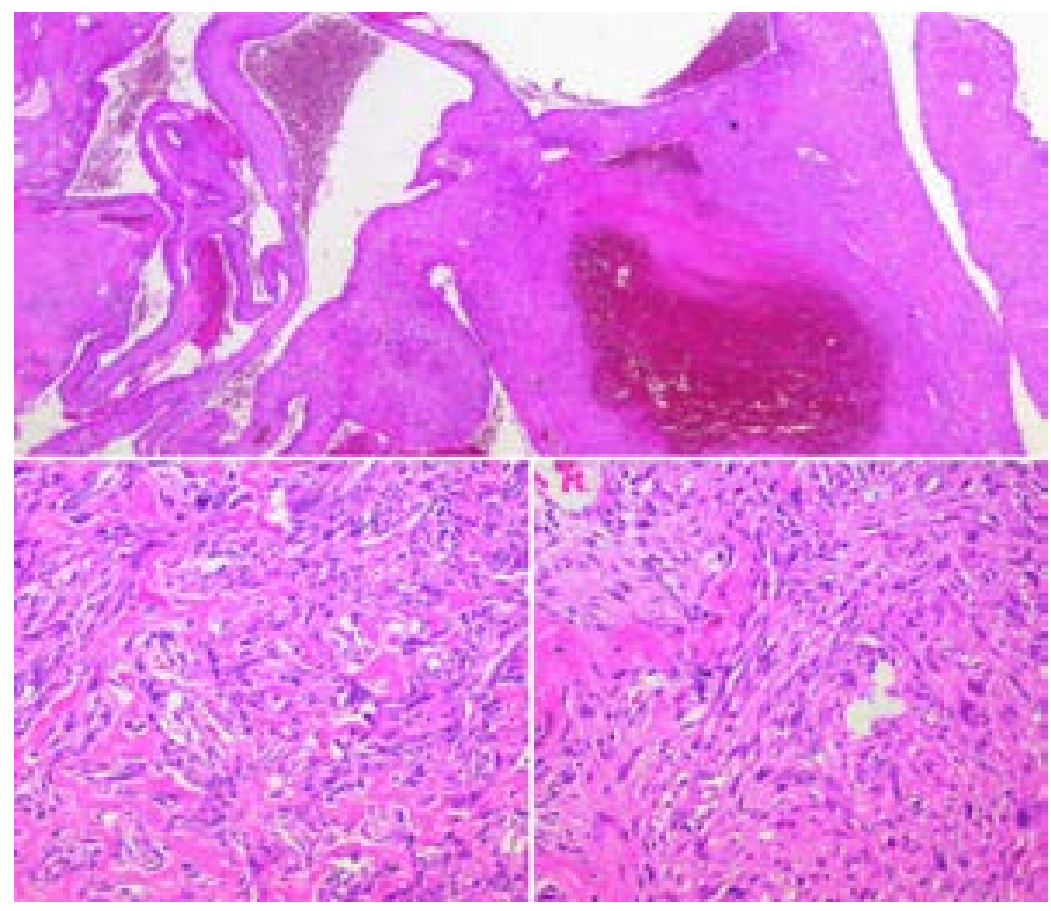

Figure 5. Top image shows cystic, blood-filled spaces characteristic of aneurysmal bone cyst (H\&E x2). Bottom images: the first image shows solid areas with osteoblastic differentiation, comprising plump, round to spindled osteoblast-like cells interspersed between delicate, lace-like trabeculae of osteoid and maturing woven bone (H\&E x20). The second image shows solid component which contained osteoclast-like giant cells and scattered larger, cytologically atypical cells (H\&E x20).

The lesion initially polarised opinion within the division. On morphology alone, the tumour showed features compatible with a diagnosis of osteoblastoma, yet also demonstrated a worrying degree of cytologic atypia and proliferative activity, which raised the diagnostic consideration of telangiectatic osteosarcoma. However, the lumbar spine is a very unusual site for osteosarcoma ${ }^{13}$, and overall the degree of atypia was considered to be insufficient for a definite diagnosis of malignancy. Telangiectatic osteosarcoma is characterised by markedly pleomorphic cells in the septa between the haemorrhagic cystic spaces, with minimal osteoid production. ${ }^{14}$ These features were absent in the tumour presented in this report. International experts on bone pathology informally reviewed the scanned histology sections, with opinion ranging between benign and malignant. Finally, the decision was taken to regard the tumour as a benign osteoblastoma with atypical features, and secondary aneurysmal bone cyst formation. The Spinal Unit was advised to manage the lesion as a benign tumour, albeit warranting careful radiological scrutiny and close follow-up.

The patient remained stable, received in-patient physiotherapy, recovered motor power fully and remained actively mobile post-surgery. She underwent screening Computed Tomography (CT) scan of the Chest as well as Technetium bone scan for other possible tumour sites following recommendation from the Sarcoma Team who were invited for their expert review of the patient. Both results were negative for other tumour deposits. She was subsequently discharged home for out-patient follow up in the Spinal Clinic in line with the recommendations of the Pathologist. The duration of hospital stay was about 
4 weeks; one week prior to surgery and 3 weeks after surgery. She remained well at last follow up 12 weeks after discharge.

\section{Discussion}

Osteoblastoma (OTB) is a rare benign tumor that accounts for approximately $0.5 \%$ to $1 \%$ of all primary bone tumors. ${ }^{2,12}$ It is most frequently found in the posterior elements with potential extension to the vertebral bodies and grossly appears as a circumscribed, friable and hemorrhagic mass which often forms extraskel et al bone in the soft tissue. ${ }^{2,4,12}$ It presents clinically with worsening pain, neurological deterioration and scoliosis, and surgery is aimed at complete resection and preservation of surrounding neural structures. ${ }^{2,3,12}$ Histologically, osteoblastoma is identical to osteoid osteoma, but osteoid osteoma is usually smaller than osteoblastoma such that lesions larger than $2 \mathrm{~cm}$ are usually categorized as osteoblastomas. ${ }^{2,12}$ Malignant transformation have been reported including recurrence rates of up to $10 \%$ after surgical resection. ${ }^{3,12}$

Aneurysmal bone cysts $(\mathrm{ABC})$ are also rare, benign lesion of bones and can be located in all bone types; frequently in femur, tibia, and small bones of the hands and feet. ${ }^{2,12}$ Approximately $8 \%$ to $30 \%$ of $\mathrm{ABCs}$ arise in the spine, mostly in the thoracic and the lumbar regions, and grossly consists of a soft fibrovascular core containing bloodfilled cyst-like cavities surrounded by a bone shell. ${ }^{2,12}$ It frequently affects the younger age below 20 years and with a slight female preponderance. ${ }^{2}$ It presents clinically with back pain, stiffness and swelling, and may also present with neurological deficit from cord/conus/equina compression and spinal instability. ${ }^{2}$ Aneurysmal bone cysts may be primary lesions developing independently or secondary lesions which can be associated with osteoblastoma, giant cell tumor, osteosarcoma, and fibrous dysplasia. ${ }^{1,2,12,15}$

The association between osteoblastoma and aneurysmal bone cyst has been reported in fronto-parietal bone and the posterior cranial fossa among other bones. ${ }^{1,12,16}$ This association is quite rare in the spine and more recently only two cases of osteoblastoma with co-existent aneurysmal bone cyst in children has been reported in the cervical and lumbar vertebrae., ${ }^{212}$ The clinical presentation of our case with worsening back pain, swelling and neurological deficit is similar to reports in the literature. ${ }^{2,3}$ and as with the two other reported paediatric cases by Hu et $\mathrm{al}^{2}$ and Pavanello et $\mathrm{al}^{12}$, the lesion extends to the vertebral body and posterior elements of affected vertebra. However, the authors wish to highlight the diagnostic challenges faced with this case both radiologically (which considered possible differential diagnoses consisting of aneurysmal bone cyst, giant cell tumour, chondroblastoma or osteoblastoma) and most especially pathologically. Histologically, similar features are displayed by different primary spinal bone tumours making diagnosis challenging. Telangiectatic Osteosarcoma mimics aneurysmal bone cyst or giant cell tumour radiologically. ${ }^{17,18}$ Generally, in cases under the age of 18 years, differential diagnosis for benign primary spinal bone tumours should include hemangiomas, eosinophilic granuloma, osteoid osteoma, osteoblastoma, aneurysmal bone cyst and giant cell tumour, while consideration for malignant tumours in children should be osteosarcoma and Ewing's sarcoma. ${ }^{19}$ The lesion in our patient radiologically showed two different mass lesions which consisted of a lesion in the L3 vertebra with no fluid-fluid levels and the lobulated lesion posterior to the vertebral bodies of L3, L4 and L5 with fluid-fluid levels (Fig. 3), hence radiologically pointing to possibility of two different lesions. Although the case was also a diagnostic challenge to the pathologists, a final diagnosis of osteoblastoma with co-existent aneurysmal bone cyst was accepted to guide therapeutic considerations and follow-up after surgical excision.

Spinal instability and neurological compromise are the foremost and grave problems in patients with tumours of the spinal column. ${ }^{19}$ These make the treatment of primary spinal tumours quite challenging in children who, unlike adults, have not achieved complete skeletal growth, and which must be taken into account when considering treatment, in addition to the other factors of spinal stability, surgical versus non-surgical interventions and preservation of neurological function. The gold standard of treatment is complete surgical excision of both osteoblastoma and $\mathrm{ABC}$ s to offer the best chance for cure and reduce the risk of recurrence., ${ }^{2,12}$ And since spinal instability can be caused by both the lesion itself and the effect of surgical removal of the lesion, this should be prevented by performing an arthrodesis. ${ }^{212}$ The high risk of massive primary haemorrhage associated with surgical excision of ABCs, which was evident in our patient, can be reduced by pre-operative embolization of $\mathrm{ABCs} .^{12}$ 
To prevent iatrogenic instability, our patient had instrumented stabilization and fusion planned as part of the surgical management of the tumour. However, unlike $\mathrm{Hu}$ et $\mathrm{al}^{2}$ and Pavanello et $\mathrm{al}^{12}$ who used both anterior and posterior approaches to achieve spinal stabilization, only posterior approach was used in our patient. The patient remained pain free and actively mobile with no neurological deficit post-operatively.

\section{Conclusion}

The case highlights the diagnostic challenges with this rare tumour and the significant role of surgical management via spinal stabilization and fusion after gross total tumour excision, which offers the best chance of cure and reduction of the risk of recurrence. However, longterm follow-up is necessary for early detection of any tumor recurrence and spinal deformity.

\section{Disclosure of conflict of interest}

None.

\section{References}

1. Kubota Y, Mitsukawa N, Arikawa R, Akita S, Satoh K. Fronto-parietal osteoblastoma with secondary aneurysmal bone cyst: A case report. Journal of Plastic, Reconstructive \& Aesthetic Surgery. 2013;66:270-273.

2. Hu H, Wu J, Ren L, Sun X, Li F, Ye X. Destructive osteoblastoma with secondary aneurysmal bone cyst of cervical vertebra in an 11-year-old boy: Case Report. Int J Clin Exp Med. 2014;7:290-295.

3. Avadhanam PK, Vuyyur S, Panigrahi KM. A rare occurrence of osteoblastoma in a child. J Pediatr Neurosci. 2010;5:153-156.

4. Mujagić S, Kuljanin M, Hrustić A. A spinal osteoblastoma in a child, situated in the vertebral body - CT and MR imaging. Paediatrics Today. 2016;12:97-101.

5. Lucas DR, Unni KK, McLeod RA, O’Connor MI, Sim FH. Osteoblastomas: clinicopathologic study of 306 cases. Hum Pathol. 1994;25:117-34.

6. Ortmann F. Osteoblastoma. eMedicine. Sep 15, 2014 http://emedicine.medscape.com/article/1257927-treatment\#showall (accessed 23 June, 2016).

7. Sama AA. Spinal Tumors. eMedicine Dec 11, 2014 http://emedicine.medscape.com/article/1267223-overview\#showall (accessed 23 June, 2016).
8. Abdel Razek AA, Castillo M. Imaging appearance of primary bony tumors and pseudo-tumors of the spine. $J$ Neuroradiol 2010;37:37-50.

9. Cugati G, Pande A, Jain PK, Symss NP, Ramamurthi $\mathrm{R}$, Vasudevan CM. Aneurysmal bone cyst of the lumbar spine. Asian J Neurosurg 2015;10:216-218.

10. Khalil IM, Alaraj AM, Otrock ZK, Chamoun RB, Sabbagh AS, Skaf GS. Aneurysmal bone cyst of the cervical spine in a child: case report and review of the surgical role. Surgical Neurology. 2006;65:298-303.

11. Dekeuwer P, Odent T, Cadilhac C, Journeau P, Langlais J, Padovani JP, Glorion C, Pouliquen JC. [Aneurysmal bone cyst of the spine in children: a 9-year follow-up of 7 cases and review of the literature]. Rev Chir Orthop Reparatrice Appar Mot. 2003;89:97-106.

12. Pavanello M, Melloni I, Fiaschi P, Consales1 A, Piatelli1 G, Ravegnani1 M, Nozza P, Gandolfo C, Cama A. A rare case of osteoblastoma associated to aneurysmal bone cyst of the spine. Case report. British Journal of Neurosurgery. 2016;30:106-109

13. Ilaslan H, Sundaram M, Unni KK, Shives TC. Primary vertebral osteosarcoma: imaging findings. Radiology. 2004;230:697-702

14. Huvos AG, Rosen G, Bretsky SS, Butler A. Telangiectatic osteogenic sarcoma: a clinicopathologic study of 124 patients. Cancer. 1982;49:1679-89

15. Zhipeng Wu, MD, Xinghai Yang, MD, Jianru Xiao, MD, DO, Dapeng Feng, MD, Quan Huang, MD, Wu Z, Yang X, Xiao J, Feng D, Huang Q, Zheng W, Huang W, Zhou Z. Aneurysmal Bone Cyst Secondary to Giant Cell Tumor of the Mobile Spine A Report of 11 Cases. Spine. 2011;36:1385-1390

16. Han X, Dong Y, Sun K, Lu Y. A huge occipital osteoblastoma accompanied with aneurysmal bone cyst in the posterior cranial fossa. Clinical Neurology and Neurosurgery. 2008;110:282-285.

17. Katonis P, Datsis G, Karantanas A, Kampouroglou A, Lianoudakis S, Licoudis S, Papoutsopoulou E, Alpantaki K. Spinal Osteosarcoma. Clinical Medicine Insights: Oncology. 2013;7:199-208.

18. Liu J, Liu S, Wang J, Zhu W, Hua Y, Sun W, Cai Z. Telangiectatic osteosarcoma: a review of literature. Onco Targets and Therapy. 2013;6:593-602

19. Ciftdemir M, Kaya M, Selcuk E, Yalniz E. Tumours of the Spine. World J Orthop 2016;7:109-116. 